\title{
Alpha-cells: Its Role as the Islet Harmonizer
}

\author{
Nabil Kamal Elnaggar ${ }^{1}$, Mohamed Nabil Elnaggar ${ }^{2}$ \\ ${ }^{1}$ Diabetes, Obesity and Endocrinology Center, HaiAljamea Hospital, Jeddah, Kingdom of Saudi Arabia \\ ${ }^{2}$ Department of Endocrinology, Diabetes \& Metabolism, University Hospitals of Morecambe Bay NHS Foundation Trust, Lancaster, United \\ Kingdom
}

Email address:

Nelnaggar@yahoo.com (N. K. Elnaggar),mnkelnaggar@gmail.com (M. N. Elnaggar)

\section{To cite this article:}

Nabil Kamal Elnaggar, Mohamed Nabil Elnaggar. Alpha-cells: Its Role as the Islet Harmonizer. International Journal of Diabetes and Endocrinology. Special Issue: Hypoglycemia in Diabetes. Vol. 4, No. 4, 2019, pp. 104-107. doi: 10.11648/j.ijde.20190404.13

Received: October 16, 2019; Accepted: November 18, 2019; Published: November 27, 2019

\begin{abstract}
It has long been thought that the alpha cells and its secretory products play an important role solely in maintaining euglycemia and preventing hypoglycemia through a contradictory action to the B cell's insulin. The $\alpha$-cell function is tightly regulated by various physiological inputs including systemic energy status, central and autonomic nervous systems, and the endocrine system. It is also an important amino acid sensor, glucagon blockade suppresses hepatic amino acid catabolism and increases the serum amino acid level. In addition to those controllers, the intra-islet microenvironment, where $\alpha$-cells are located, has been recently revealed to be important in the regulation of the various cellular secretory functions including the overlapping of glucagon and insulin secretion through a precise cell-cell crosstalk. Paracrine interactions between pancreatic islet cells have been proposed as a mechanism to regulate hormone secretion and glucose homeostasis, alpha and B cells are closely positioned on the sides of their blood supply where acetylcholine acts as the paracrine communicator of signals inside the islets. Recently, it has been demonstrated that blocking acetylcholine esterase increases insulin secretion. Moreover, it has also been suggested that glucagon is not exclusively a counter-regulatory hormone that elevates blood glucose levels, in contrast it can cause hypoglycemia conditioned by the presence of intact B cells and a functional GLP-1R (glucagon-like peptide 1 receptor). These data argue for glucagon agonism in modern management of T2DM. Alpha-cells also, have been shown to be able to trans-differentiate into $\beta$-cells only in the presence of insulin-positive cells with $\alpha$-cell origin in the lineage tracing analyses, confirming the role of $\alpha$-cells as a source of $\beta$-cell regeneration. The article reviews the updated knowledge about the functions of the alpha-cells and its role in the paracrine control of islet cell secretions and the future therapeutic potentials.
\end{abstract}

Keywords: Pancreatic Islet Cells, B Cells, Alpha Cells, Glucagon, GIP (Glucose-dependent Insulinotropic Polypeptide), GLP-1 (Glucagon-like Peptide 1)

\section{Introduction}

Over nine decades ago the alpha cells were identified as the source of the hyperglycemia producing "contaminant" factor contained in the pancreas extracts used to isolate insulin. The hyperglycemic factor was named glucagon and subsequently shown to arise from alpha cells that co-occupy the islets of Langerhans in the pancreas along with the beta cells that produce insulin, and accompanying delta and PP cells that produce the hormones somatostatin and pancreatic polypeptide, respectively. As a consequence of its glucose-elevating actions, glucagon was proposed 45 years ago to be diabetogenic, an important contributor to the fasting hyperglycemia that occurs in patients with diabetes.
However, recently there is much interest in the central roles of glucagon in the development of diabetic hyperglycemia and this revival of glucagon theory, also enhanced the progress of basic research in alpha-cell biology, as targeting glucagon is now expected to be the next therapeutic approach in diabetes treatment [1-3].

\section{Mechanisms of Islet Cell Control}

Looking deeper into the mechanisms that control alpha cell glucagon secretion revealed that besides the central nervous and autonomic control and other endocrine hormonal control; the impact of the intra-islet microenvironment on the harmony of islet cells hormonal secretions was not yet much appreciated. The anatomical analyses of human pancreatic 
islets show a tight positional association between alpha-, beta and delta cells that enables their communication through their secretions in a paracrine manner (figure 1). In other words, a-cell function, such as glucagon secretion, is regulated in an intra-islet manner by inputs from other endocrine cells, including insulin from beta cells and somatostatin from D-cells. Meanwhile alpha-cells certainly influence their neighboring cells through various outputs. Indeed, glucagon and acetylcholine, both secreted from alpha cells in human islets, stimulate insulin secretion, whereas all known secretory products of beta cells inhibit glucagon secretion [4-6].

\section{The Alpha Cell Extended Functions and the Paracrinopathy Effects}

The alpha cell is not only a hyperglycemic gland but in a recent study [6], it was found that pancreatic a-cell function independently contributed to insulin sensitivity, even in patients with T1DM, whose B-cell function is diminished probably through their role in secreting GLP-1 (glucagon-like peptide 1). The paracrine glucagon signaling in the islet is critical for the beta cell to secrete the appropriate insulin amounts that sustain the human glycemic set point. This is in line with studies showing that glucagon signaling through glucagon and GLP-1 receptors contributes substantially to the beta cell's secretory responsiveness and competence by increasing cAMP levels [7-10].

In the human islet under normoglycemic conditions ( $~ 90$ $\mathrm{mg} / \mathrm{dl})$, alpha cell activation overlaps with beta cell activation. Indeed, when isolated human islets are exposed to step increases or decreases in glucose concentration, it is clear that both insulin and glucagon secretion are stimulated at $90 \mathrm{mg} / \mathrm{dl}$. This is contrary to the general notion that glucagon and insulin secretion are mutually exclusive. Although seemingly counterintuitive because these hormones have antagonistic effects on plasma glucose levels, these findings make sense if we consider glucagon a local paracrine signal that amplifies insulin secretion to stabilize glucose levels.

Increased glucose variability is also associated with a "paracrinopathy" characterized by paradoxical behaviors of alpha-cells during glycemic changes; that is, a deficient glucagon response to hypoglycemia and an inappropriately high glucagon response to hyperglycemia.

Moreover, recent research had discovered that alpha-cells secrete other hormones such as: GLP-1, GIP (Glucose-dependent insulinotropic polypeptide), and acetylcholine. Hyperplasia of alpha cells results in the induction of the expression of $\mathrm{PC} 1 / 3$ and their production of GLP-1 in large amounts resulting in marked elevations in the circulating plasma levels of both GLP-1 and glucagon [11]. Two conditions that lead to marked alpha cell hyperplasia have been extensively described: One is injury of beta cells of diverse types and the other is impaired glucagon receptor signaling.

These recent discoveries extends alpha-cell functions to involve the continuous cross-talk between islet cells and even helping in preservation of their integrity and life span.

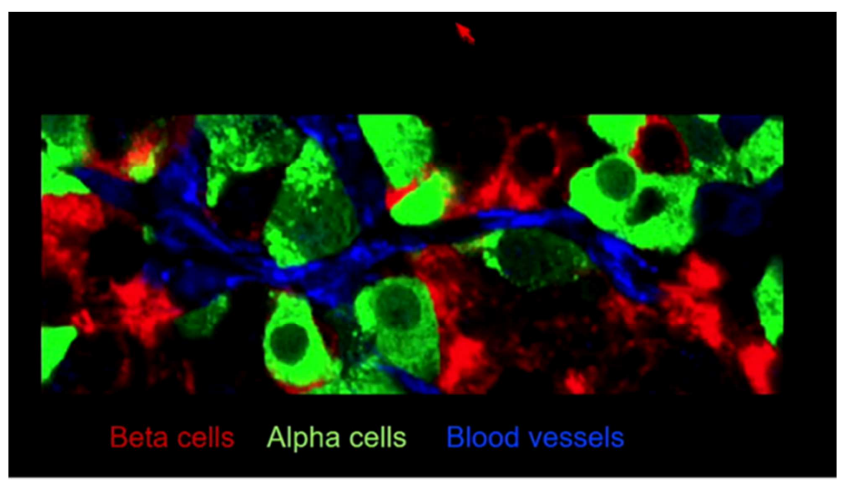

Figure 1. Arrangement of islet cells around blood vessels.

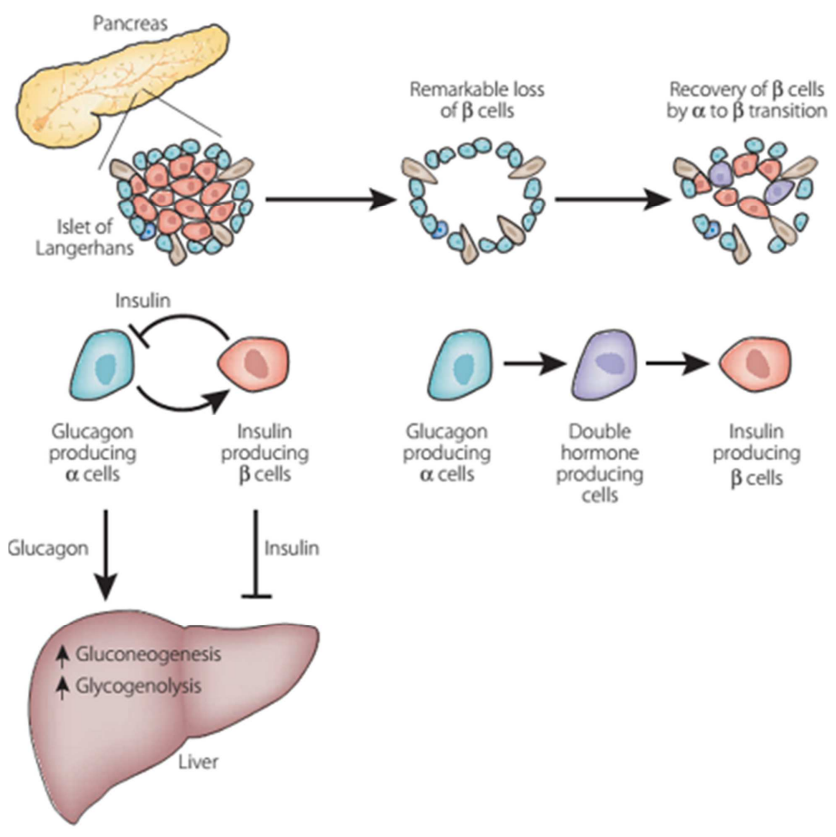

Figure 2. Alpha cell versatility.

\section{Alpha Cells: The Versatile}

The islet beta cells were shown to differentiate into alpha cells in response to prolonged glucotoxicity and long duration diabetes; in more than one study hyperglycemia-induced glucotoxic stress dedifferentiated mouse beta cells into Ngn3 positive cells that no longer express insulin [1-5]. Remarkably, upon normalization of plasma glucose levels and resulting alleviation of glucotoxic stress, these same undifferentiated Ngn3 positive cells redifferentiate back into functioning beta cells that produce insulin and thereafter maintain euglycemia [11].

It was also very interesting to discover that the reverse is possible, specifically immature alpha cells, pro-alpha cells, are precursors of beta, meaning that the alpha cells can transdifferentiate into b-cells by the presence of insulin-positive cells with a-cell origin in lineage tracing analyses, confirming the role of a-cells as a source of b-cell regeneration and pointing to their capacity to control the islets mass in both b-cells and themselves. DAI X, et al [3], found 
distinct $\alpha$-cell sub-populations in human islets that are either resistant to, or sensitive to, dysfunction in the form of a shift towards a more ' $\beta$-cell like' functional phenotype in T2D.

Key studies in genetically engineered mice demonstrated that the induction of Pax4 overexpression (by tamoxifen) in alpha cells resulted in the highly efficient transdifferentiation of alpha cells into beta cells (Figure 2)

This transdifferentiation severely depleted the population of alpha cells, thereby lowering plasma levels of glucagon, resulting in the stimulation of proliferation of duct-associated stem/progenitor cells that differentiated into immature pro-alpha cells. These findings of reversible dedifferentiation

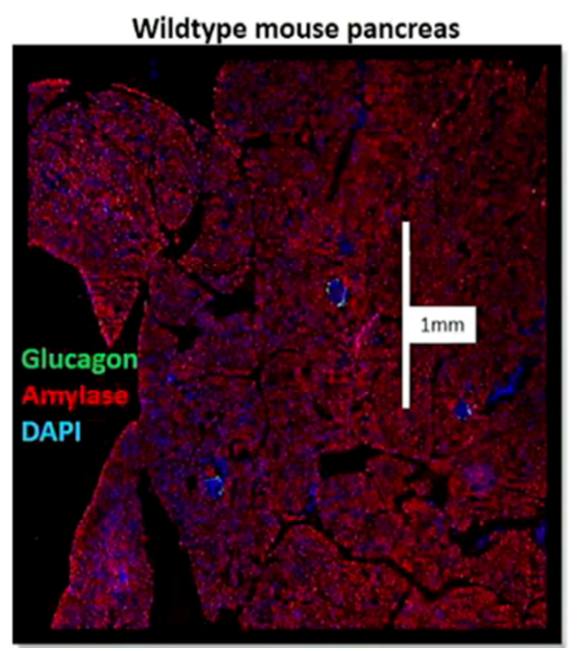

and redifferentiation of $\mathrm{B}$ cells demonstrate the remarkable plasticity of islet endocrine cells [4-5].

\section{Potential the Rapeutic Implications}

Antagonising glucagon seemed to be a good solution for the unsuppressed glucagon production, however, the Gcg null and Gcgr null mice developed massive alpha cell hyperplasia. As the Gcg null mice age, $100 \%$ of them develop pancreatic neuroendocrine tumors that are malignant and metastasize to liver and brain, ending up the promise of their role in therapy [12-14], (Figure 3).

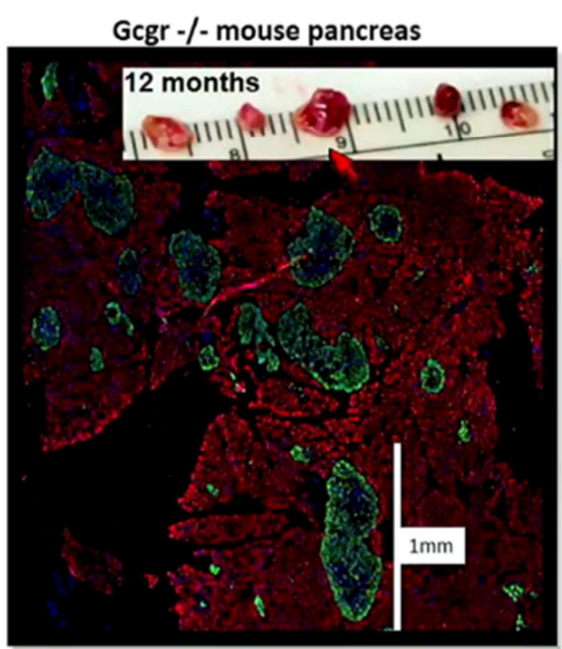

Figure 3. Effects of Gcr knock out in mice.

Appreciating the harmony of secretion and function among the three hormones: glucagon, GIP and GLP-1, highlighted the importance of evaluating balanced signaling at the targeted receptors. The development of intermixed, unimolecular peptides with activity at each of their respective receptors has been a new area of research and innovation.

The biological activity of these multireceptor agonists are founded in the success of GLP-1-directed strategies; by including GIP and glucagon components, these multireceptor agonists are thought to enhance GLP-1's activities by broadening the tissue targets and synergizing at tissues that express multiple receptors, such at the brain and islet b cells [15].

\section{Summary}

The findings of intimate cell-cell contacts between alpha and beta cells in the islets suggest the existence of intercellular paracrine communication between these two cell types. One function of alpha cells is to induce the production of GLP-1 in response to injury signals emanating from stressed beta cells. GLP-1 is known to provide cytoprotection for and to stimulate the proliferation of beta cells. The information gathered on the functions of alpha cells supports its important role for the protection and regeneration of beta cells. It is tempting to conjecture that because of the critical importance of insulin produced by beta cells both in development and in adult life, alpha cells have evolved to function as helper cells to maintain the health of beta cells. Furthermore, in conditions of severe depletion of beta cells, alpha cells are prompted to transdifferentiate into new beta cells to replace those that are lost.

\section{References}

[1] Kawamori D. Exploring molecular mechanisms underlying aand b-cell dysfunction in diabetes. Diabetol Int 2017; 8: 248256.

[2] Stanojevic V, Habener JF. Evolving function and potential of pancreatic alpha cells. Best Pract Res Clin Endocrinol Metab 2015; 29: 859-871.

[3] Xiaoqing Dai, et al. Pancreatic Alpha-Cell Function and Identity in Human T2D. Diabetes 2018 Jul; 67 (Supplement 1): https://doi.org/10.2337/db18-315-LB

[4] Kawamori D. Alpha the versatile: Guardians of the islets. J Diabetes Investig. 2019 Jan; 10 (1): 26-28. doi: 10.1111/jdi.12875. Epub 2018 Jul 25.

[5] Lam CJ, Cox AR, Jacobson DR, et al. Highly proliferative alpha-cellrelated islet endocrine cells in human pancreata. Diabetes 2018; 67: 674-686.

[6] Takahashi $\mathrm{N}$ et al. Contribution of pancreatic a-cell function to insulin sensitivity and glycemic variability in patients with type 1 diabetes. J Diabetes Investig Vol. 10 No. 3 May 2019. 
[7] Bertuzzi F, Berra C, Socci C, Davalli AM, Calori G, Freschi M, Piemonti L, De Nittis P, Pozza G, Pontiroli AE. Glucagon improves insulin secretion from pig islets in vitro. J Endocrinol. 1995; 147: 87-93. [PubMed: 7490541].

[8] Huypens P, Ling Z, Pipeleers D, Schuit F. Glucagon receptors on human islet cells contribute to glucose competence of insulin release. Diabetologia. 2000; 43: 1012-1019. [PubMed: 10990079].

[9] Pipeleers D, Veld PI, Maes E, and Van De Winkel M. Glucose-induced insulin release depends on functional cooperation between islet cells. Proc Natl Acad Sci U S A. 1982; 79: 7322-7325. [PubMed: 6760195]

[10] Samols E, Marri G, Marks V. Promotion of insulin secretion by glucagon. Lancet. 1965; 2: 415-416. [PubMed: 14346763]
[11] Rodriguez-Diaz $\mathrm{R}$ et al., paracrine interactions within the pancreatic islet determine the glycemic set point. Cell Metab. 2018 March 06; 27 (3): 549-558.e4. doi: 10.1016/j.

[12] Holst JJ. The physiology of glucagon-like peptide 1. Pysiol Rev. 2007 Oct; 87 (4): 1409-39.

[13] Vishal Gupta. Glucaon-like peptide 1 analogues: An overview. Indian J Endocrinol Metab. 2013 May-Jun; 17 (3): 413-421.

[14] Prashant Nadkarmi, Oleg G. Chepurny, and George G. Holz. Regulation of Glucose Homeostasis by GLP-1. Prog Mol Biol Trans Sci. 2014; 121: 23-65.

[15] Megan E. Capozzi, 1 Richard D. DiMarchi, Matthias H. Tschop, BrianFinan, and Jonathan E. Campbell. Targeting the Incretin/Glucagon System WithTriagonists to Treat Diabetes. Endocrine Reviews, October 2018, 39 (5): 719-738. 